\title{
CAMBIO CONCEPTUAL Y METODOLÓGICO EN LA ENSEÑANZA Y EL APRENDIZAJE DE LA EVOLUCIÓN DE LOS SERES VIVOS. UN EJEMPLO CONCRETO
}

GENÉ, A.

Departament de Didàctica de la Matemàtica i de les Ciències Experimentals. Universitat Autònoma de Barcelona. Camp de Mart, 33, 25004 - Lleida

\section{SUMMARY}

In this work we developed and assessed the efficiency of a didactic lesson based on the science teaching-learning paradigm as a conceptual, methodological and attitudinal change.

For this purpose we chose the topic of the evolution of living beings through natural selection.

\section{INTRODUCCIÓN-PLANTEAMIENTO DEL PROBLEMA E HIPÓTESIS DE TRABAJO}

Desde el trabajo de Lucas (1971), en el que por primera vez se constata la similitud entre las explicaciones de los alumnos respecto a la evolución de los seres vivos con las de Lamarck, las investigaciones sobre las preconcepciones referidas a este tema han ido aumentando. Se ha mostrado la coincidencia de estas ideas en estudiantes de muchos países lejanos entre sí, tanto geográfica como culturalmente. Así, Jungwirt (1975) encuentra ideas lamarckianas en escolares de Israel; Brumbi (1980), en escolares ingleses; Hallden (1988), en adolescentes suecos; Kinnear (1983) y Martin ( 1983), en estudiantes de Australia.

En nuestro país, dos recientes trabajos (Albadalejo y Lucas 1988 y Jiménez Aleixandre 1989) han estudiado las características del pensamiento de los alumnos sobre este tema. El primero muestra las concepcions sobre Ia mutación en los estudiantes de BUP. Jiménez Aleixandre en su interesante tesis doctoral, además de estudiar las concepcions de los alumnos, propone un modelo de enseñanza / aprendizaje para salir al paso de los errores.

Nuestro trabajo no pretende mostrar que hay preconcepciones incorrectas. Nuestro estudio plantea hasta qué punto un determinado modelo didáctico puede favorecer o no la superación de estas preconcepciones.
En nuestra opinión, el hecho que los estudiantes expliquen Ias modificaciones de los seres vivos de la misma manera que lo hizo Larnarck, no puede ser debido a la casualidad. No puede explicarse por el azar. Pensamos con Gil (1983; Gil, Carrascosa, 1985) que la coincidencia de las ideas es debida a la coincidencia en la metodoIogía utilizada para aproximarse a la realidad. Una metodología que no va más allá de la evidencia, de la simple observación y de la inducción de conclusiones a partir de la misma.

La utilización de instrumentos de aprendizaje de estas características lleva a pensar que un órgano crece porque se usa mucho, y se atrofia si no se usa. Que estas características pasen de padres a hijos también es fácil de explicar, con sentido común, por la herencia de los caracteres adquiridos, como hizo Lamarck. Veamos algún ejemplo.

Planteamos a alumnos de 14 años la siguiente cuestión (Grau 1989):

Las aves acuáticas que nadan por la superficie del agua y utilizan las patas para impulsarse tienen una membrana interdigital desarrollada, como la de la fígura, que hace más eficaz el movimiento de la pata, y por tanto, su desplazamiento. 
Explica cómo crees que se ha originado esta membrana.

Respuesta escolar: "Yo pienso que las aves acuáticas van desarrollando esta membrana a lo largo de su vida hasta llegar a adultos. Quiero decir que como los patos desde pequeños están en contacto con el agua, el movimiento continuo de sus patas hace que la membrana vaya siendo cada vez más fuerte. Si no se encontraran en contacto con el agua, esta membrana desaparecería ya que no tendría ninguna utilidad. Los patos pequeños heredan de sus padres esta membrana.»

\section{Respuesta de Lamarck: Filosofia Zoológica (1809)}

«...En todo animal que no haya llegado al final de su desarrollo, el uso frecuente y sostenido de un órgano, lo fortifica, lo desarrolla lo agranda y le da una potencia proporcional a la duración de su uso. En cambio, la falta constante de uso de un órgano, lo debilita, lo deteriora, disminuye progresivamente sus facultades y acaba por hacerlo desaparecer.»

«...Todo cambio adquirido en un órgano por un hábito de uso suficiente, se conserva y pasa a las otras generaciones si el cambio es común ai macho y a la hembra....”

Siguiendo con nuestro razonamiento, si la causa de que exista coincidencia entre las ideas de Lamarck y nuestros alumnos está en la forma «natural» de acercarse a la realidad, será preciso modificar esta metodología para que superen las concepciones incorrectas. Pero, ¿en qué sentido debe modificarse la forma de acercarse a la realidad?

Un primer requisito será hacer dudar de las evidencias, hacer dudar del sentido común. Para ello se deben plantear cuestiones o situaciones que no puedan explicarse por el pensamiento del alumno, por su propio "paradigma».

El conflicto que surge con el propio pensamiento es eI punto de partida para ir construyendo nuevos conocimientos pero, eso sí, con una metodología diferente que parta de este problema-conflicto. La solución a este problema ha de estar dirigida por hipótesis de trabajo cuya contrastación vaya permitiendo destruir los errores y construir un nuevo marco conceptual, una nueva teoría.

Nuestra hipótesis de trabajo pues, se concreta en que la utilización de una unidad didáctica con estas orientaciones facilitará la superación de las concepciones incorrectas de los alumnos y la construcción de la teoría de la evolución de los seres vivos por selección natural.

Vamos a continuación a mostrar cómo ha sido contrastada esta hipótesis.

\section{DISEÑO EXPERIMENTAL}

El eje central del diseño experimental ha sido la confección de una unidad didáctica fundamentada en el modelo que se propone.
El hilo conductor de esta unidad - de la que se muestra algunos fragmentos en el anexo- ha sido la considera: ción de aspectos clave, que a nivel histórico, fueron útiles para la construcción de la teoría de la evolución por selección natural.

La unidad plantea los siguientes problemas:

1. ¿Cómo cambian los seres vivos?

2. ¿Puede variar el número de individuos de una población?

3. ¿Qué pasa cuando las condiciones ambientales cambian?

4. ¿Qué es una mutación?

5. ¿Cómo actúa el ser humano sobre les especies para sacar un beneficio?

6. ¿Por qué son tan diferentes los individuos salvajes y domésticos de una misma especie?

7. ¿Cómo se forman las nuevas especies?

8. ¿Qué les pasó a los dinosaurios?

9. ¿Dónde esta localitzado el material genético que hace que un ser vivo modifique sus características?

10. ¿Puede explicarse la evolución humana por la teoría de la evolución por selección natural? ¿Cómo se originaron las mujeres y los hombres?

11. ¿Ha influido la teoría de la evolución de los seres vivos en la sociedad humana?

La unidad didáctica se trabajó con alumnos de $8^{\circ}$ de EGB y futuros profesores de primaria.

Las actividades de la unidad pensadas para conectar con los conocimientos previos y, por tanto, para iniciar el aprendizaje, fueron utilizadas para conocer estadísticamente los preconceptos. Estos primeros resultados han sido utilizados como punto de referencia, al servir para ver hasta qué punto se había conseguido el aprendizaje significativo de la evolución de los seres vivos por selección natural.

Paralelamente se ha contado con dos grupos control que han trabajado el tema durante el mismo curso con las metodologías de trabajo de aula habituales. Una metodología (grupo c,1) responde al modelo de transmisión de conocimientos elaborados. El grupo c,2 trabajó con un modelo «activo» 0 de descubrimiento autónomo. Los resultados correspondientes a los maestros de primaria en formación se han comparado con un grupo de futuros maestros de ciencias que habían estudiado este tema en BUP y COU.

Pasamos a continuación a concretar el diseño, presentando las cuestiones utilizadas, tanto en el pretest como en el postest.

Cuestión l. Las aves acuáticas que nadan por la superficie del agua y utilizan las patas para impulsarse tienen una membrana interdigital desarrollada, como la de la figura, que hace más eficaz el movimiento de la pata y por tanto su desplazamiento.

Explica cómo crees que se ha originado esta membrana. 
Esta pregunta (Grau 1989), basada en un problema planteado por Lamarck en su libro Filosofía Zoológica, ha sido pasada como pretest a alumnos de $8^{9}$ de EGB y futuros maestros en número aproximado de un centenar.

Cuestión 2. Cuando se utilizaron por primera vez los insecticidas aerosoles contra las moscas y los mosquitos, resultaron muy eficaces, es decir, casi todos los insectos morian cuando se ponían en contacto con el insecticida.

Hoy, 30 años después, solamente una pequeña propor. ción de estos insectos muere cuando utilizamos los aerosoles con la misma composición.

Explica por qué crees que esto ocure.

Esta cuestión ha estado utilizada previamente por Jiménez Aleixandre (1989), y ha servido como postest para ver hasta qué punto se había conseguido el aprendizaje significativo de la evolución de los seres vivos por selección natural. Ha sido pasada a los grupos control, y a los alumnos de $8^{\circ}$ de EGB y futuros maestros.

A continuación vamos a presentar los resultados obtenidos.

\section{RESULTADOS-ANÁLISIS Y CONCLUSIONES}

Cuestión 1: Pretest. Las aves acuáticas que nadan por la superficie del agua y utilizan las patas para impulsarse tienen una membrana interdigital desarrollada, como la de la figura, que hace más eficaz el movimiento de la pata y, por tanto, su desplazamiento.

Explica cómo crees que se ha originado esta membrana.

Alumnos que dan una respuesta lamarckista

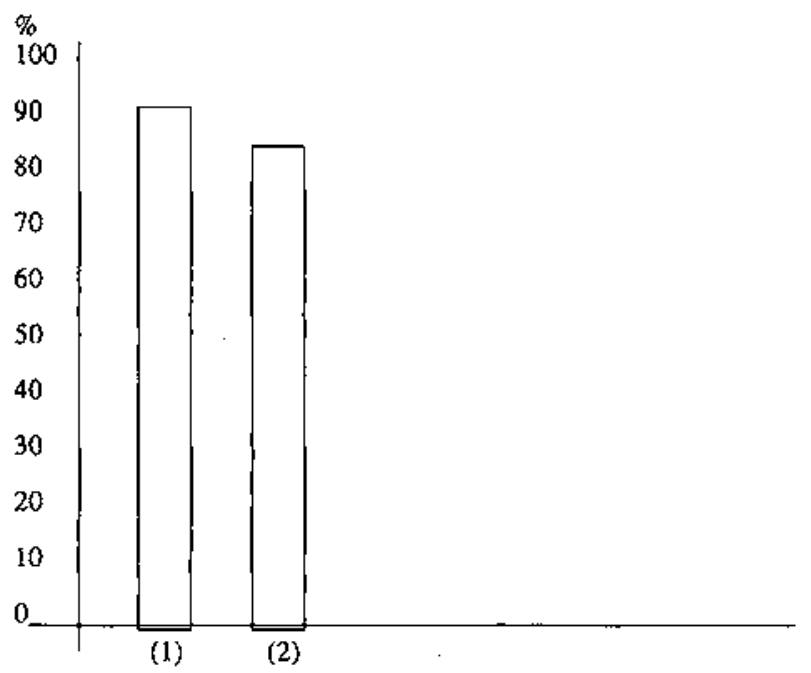

(1) Alumnos de $8^{2}$ de EGB antes de empezar a trabajar el tema con el modelo de enseranza / aprendizaje que se propone. $\mathrm{N}=30 ; \%=92 \% 3$; $s d=4$ ' 8 .

(2) Futuros maestros de primaria antes de empezar a trabajar ei tema con el modelo de enseñanza / aprendizaje que se propone. $N=54$; $\%=8 S^{\prime} 1 ; \mathrm{sd}=4^{\prime} 8$.
Cuestión 2: Postest. Cuando se utilizaron por primera vez los insecticidas aerosoles contra las moscas y los mosquitos resuitaron muy eficaces, es decir, casi todos los insectos morían cuando se ponían en contacto con el insecticida. Hoy, 30 años después, solamente una pequeña proporción de estos insectos muere cuando utilizamos los aerosoles con la misma composición.

Explica por qué crees que esto ocurre.

Alumnos que dan la respuesta correcta

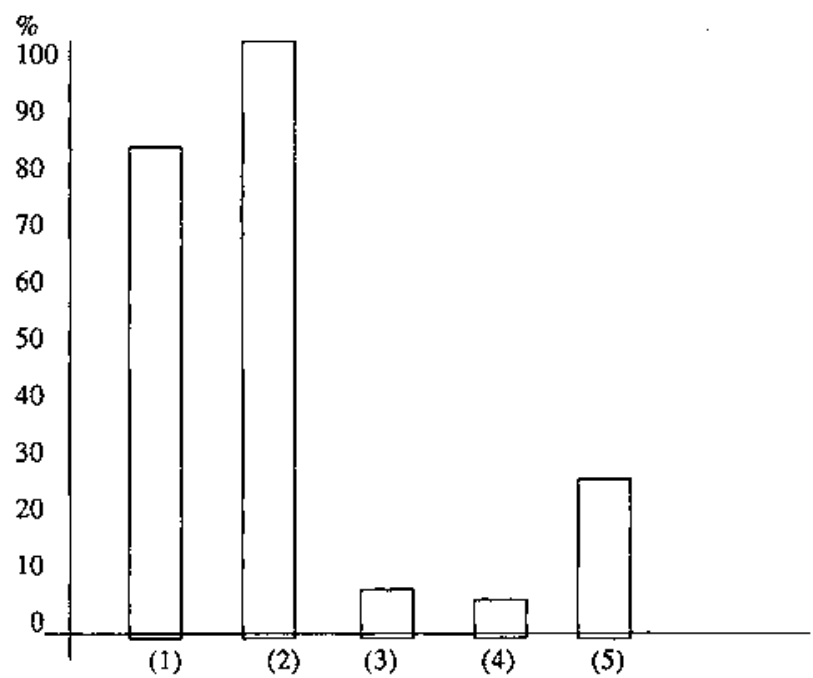

(1) AIumnos de $8^{\circ}$ de EGB que han estudiado el tema. $\mathrm{N}=30 ; \%=857$; sd $=6{ }^{\prime} 6$.

(2) Maestros en formación que han estudiado el tema. $N=54 ; \%=100$; $\mathrm{sd}=0$.

(3) Grupo de $8^{2}$ de EGB 1,c. Metodología de transmisión. $N=64 ; \%=$ 4'6; sd $=2$ ' 6 .

(4) Grupo de $8^{2}$ de EGB 2,c. Metodología de descubrimiento autónomo. $N=30 ; \%=3 \cdot 8 ; s d=34$.

(5) Maestros de ciencias en formación. Han estudiado el tema en BUP y COU. $N=\$ 1 ; \%=26^{\star} 3 ; \mathrm{sd}=6^{\prime} 1$.

La te Student nos da, comparando los grupos de $8^{\circ}$ de EGB entre sí y los grupos de futuros maestros, diferencias significativas muy elevadas, con una \& superior en todos los casos al 0'01

A la luz de estos primeros resultados, podemos pensar que el modelo de enseñanza / aprendizaje de las ciencias naturales como cambio conceptual y metodológico que se concreta en el programa-guía que presentamos en el anexo, parece ser útil para la superación de los errores conceptuales en el tema de la evolución de los seres vivos. De hecho, los alumnos de $8^{\circ}$ de EGB y los futuros maestros aprendieron de forma significativa la evolución de los seres vivos por selección natural.

Hemos de indicar que a los alumnos de $8^{\circ}$ de EGB, que utilizaron el modelo que proponemos se les planteó, después de tres meses de terminado el tema, dos problemas para ver si en sus soluciones aplicaban adecuada- 
mente la teoría de la evolución por selección natural. En ambos problemas las respuestas correctas superaron el $80 \%$.

Estos resuitados parecen ser esperanzadores respecto a la eficacia del material didáctico utilizado, si bien deberemos profundizar en las causas que provocan que un $20 \%$ de alumnos hayan dado todavía explicaciones lamarckianas. Será preciso, pensamos, dar respuesta a cuestiones como:

$-i$ De qué manera se puede afrontar en el aula y en el centro las dificultades que tienen algunos alumnos y alumnas para realizar un cambio conceptual y metodológico?

- ¿Hasta qué punto instrumentos básicos de aprendizaje como: la lectura comprensiva de textos, la realización de resúmenes, la capacidad para realizar debates, de expo- ner con claridad y concreción las ideas... puede facilitar este cambio?

- ¿Se puede facilitar desde la enseñanza primaria el cambio metodológico?

- ¿Cómo relacionar la enseñanza de las ciencias en la escuela primaria con la secundaria para favorecer este cambio?

- Etcétera.

NOT A: Este trabajo ha sido realizado con la ayuda del Institut d’Estudis Ilerdencs de Lleida.

\section{REFERENCIAS BIBLIOGRÄFICAS}

ALBADALEJO, C. y LUCAS, A., 1988. Pupils' meaning for mutation. Journal of Biological Education, 22(3), pp. 215-219.

BRITSH MUSEUM (1981)/ DIPUTACIÓ DE BARCELONA (1982). L'Origen de les Espècies.

BRUMBI, M., 1984. Misconceptions about the concept of Natural Selection by Medical Biology Students, Science Education, 68 (4), pp. 493-503.

GIL, D., 1983. Tres Paradigmas básicos en la Enseñanza de las Ciencias, Enseñanza de las Ciencias, 1(1), pp. 26-33.

GIL, D. y CARRASCOSA, 1985. Science Learning as a Conceptual and Methodological Change, European Journal of Science Education; Vol. 7(3).

GRAU, R., 1989. Evolució dels éssers vius. Unitat didàctica (no publicado).

HALLDEN, O., 1988. The Evolution of Species: pupils perspectives and school perspectives, International Journal of Science Education, 10(5), pp. 541-552.
JIMÉNEZ ALEIXANDRE, M.P., 1989. Los esquemas conceptuales sobre la selección natural: Análisis y propuestas para un cambio conceptual. Tesis Doctoral. Universidad Complutense de Madrid.

IUNGWIRTH, E., 1975. The problem of teleology in Biology as a problem of Biology - teacher Education, Journal of Biological Education, 9 (6), pp. 243-246.

KINNEAR, J, 1983. Identification of Misconceptions in Genetics and the use of computer simulation in their correction, International Seminar on Misconceptions in Science and Mathematics. Cornell, pp. 83-92. (Helm \& Novak Ed.).

LUCAS, A.M., 1971. The teaching of «adaptation», Journol of Biological Education, 5, pp. 86-90.

MARTIN, M., 1983. An examination of estudents misconceptions in genetics, International Seminar on Misconceptions in Science and Mathematics, Comell, pp. 218-225. (Helm \& Novak Ed.). 


\section{ANEXO}

En este anexo presentamos a modo de ejemplo algunas actividades del programa-guía disefiado para trabajar el tema de la evolución de los seres vivos por selección natural.

Hemos escogido tres apartados de la unidad:

- El apartado 2 ¿Puede variar el número de individuos de una población?, tiene como objetivo ayudar a los alumnos a definir y comprender el problema que nos ocupa.

- El apartado 3 ¿Qué pasa cuando las condiciones ambientales cambian? forma parte del bloque destinado a la construcción de nuevos conocimientos.

- EI apartado 10 ¿Puede explicarse la evolución humana por la teoría de la evolución por selección natural? ¿Cómo se originaron las mujeres y los hombres? réne actividades de aplicación de los conocimientos adquiridos y de reflexión sobre el papel de la cultura en la evolución humana.

Las actividades se presentan acompañadas de unos comentarios para facilitar la aplicación en el aula.

\section{2. ¿PUEDE VARIAR EL NÚMERO DE INDIVIDUOS DE UNA POBLACION?}

\section{Actividad 1}

Los elefantes se reproducen muy lentamente, a pesar de ello en 700 años una pareja de elefantes podría haber tenido 19.000 .000 de descendientes.

Una elefanta puede tener la primera cría a los doce años, y tener otra cada dos años. Si las crías sobreviven todas y tienen cachorros y todos sobreviven y se reproducen cada dos años... podríamos explicar fácilmente estos resultados.

Compara las dos imágenes que muestran la misma zona de una sabana africana natural inalterada por donde no han pasado nunca los humanos. La primera imagen es de hace 100 años. La segunda es de nuestros días.

Emitir y fundamentar hipótesis que permitan explicar esta situación.

(NOTA: Se muestran aquí dos dibujos en los que aparece la misma zona de la sabana con prácticamente el mismo número de elefantes a pesar de que hay entre las dos escenas una diferencia de 100 años)

Comentario: Es necesario insistir en que los seres humanos no han intervenido en ningún momento en la vida de esta sabana, ya que así se fuerza a los alumnos a dar explicaciones naturales al problema que se plantea. En general, se refieren en sus hipótesis a las condiciones adversas: falta de alimento, escasez de agua, presencia de muchos depredadores, epidemias... Indican también que, cuando esto ocurre, los individuos que primeró mueren son los más débiles.

El problema que sugieren sus hipótesis es por qué un determinado individuo es mts débil que otro de su misma especie y, por tanto, tiene mayores probabilidades de ser eliminado por los factores naturales. También preguntan qué es lo que determina que un individuo sea más fuerte que otro $y$, en consecuencia, con mayores posibilidades de resistir a las condiciones adversas.

Antes de abordar este problema, clave para la comprensión de la teoría de la evolución por seleción natural, pensamos que es necesario que los alumnos comprendan que esto ocurre en todas las especies y que además el factor humano puede jugar también su papel. Para ello proponemos la realización de la siguiente actividad:

\section{Actividad 2}

Pensad ejemplos concretos de especies de animales y plantas en las que:

1) el número de individuos vaya disminuyendo

2) el número de individuos aumente

3) eI número de individuos sea prácticamente constante.

Indicar las causas que, en vuestra opinión, están contribuyendo a su disminución, aumento o equilibrio.

Contrastar vuestras opiniones con bibliografía.

Comentario: Esta actividad favorece el trabajo autónomo de los pequeños grupos. Es preciso, sin embargo, discutir la estructura del trabajo y exigir concreción en las conclusiones.

Una manera de hacerlo es organizar una mesa redonda en la que cada grupo pueda hablar durante un tiempo limilado. Después de las exposiciones se puede abrir el debate para proponer mejoras, opiniones contrarias, críticas...

Después de esta actividad, vamos a recuperar el problema que se suele plantear en la actividad 1 , o bien lo presentamos nosotros.

\section{Actividad 3}

¿Qué determina que un individuo sea más débil o más fuerte y que, en consecuencia, sea eliminado por la naturaleza o pueda sobrevivir?

Emitir hipótesis que permitan dar respuesta a esta cuestión.

Comentario: Aquí surgen interrogantes sobre el papel de la herencia por sí misma. También sobre la relación entre el ambiente y la herencia: ¿Puede el ambiente actuar favoreciendo una determinada característica de un ser vivo? ¿Quién actúa primero, la herencia o el ambiente?...

Las hipótesis generales que se plantean en los grupos y a lo largo de la puesta en comin para dar respuesta a estas cuestiones nos empiezan a aproximar a la tesis defendida por Darwin. Queda todavía un largo camino.

\section{3. ¿QUÉ PASA CUANDO LAS CONDICIONES AM- BIENTALES CAMBIAN?}

\section{Actividad 1}

Las mariposas geometras son muy abundantes en la Gran Bretaña. Bastantes especies de pájaros se alimentan de estas mariposas, que cazan cuando se encuertran descansando en las cortezas de los árboles durante el día.

Antes de 1850 casi todas las mariposas geómetras de la ciudad de Manchester eran de un color claro que se confundían muy bien con las cortezas de los árboles en los que descansaban. Una colección de mariposas geometras recogida en aquella época, tenía el siguiente aspecto. 
(NOTA: Se muestra aquí un dibujo de una colección de mariposas con una de ellas de color oscuro. Todas las demás son grises.)

A lo largo del siglo XIX, el ambiente de la ciudad de Manchester cambio radicalmente. Antes de la revolución industrial las cortezas de los árboles donde descansaban las mariposas geometras eran de color claro porque tenían unos pequeños líquenes incrustados en ellas. La contaminación producida por las fábricas a finales del siglo, mató todos estos líquenes y oscureció las cortezas.

Una colección de mariposas geometras recogida a principios del siglo XX tenía este aspecto.

(NOTA: Se muestra aquí un dibujo de una colección de mariposas con una de ellas de color gris. Todas las demás son oscuras.)

¿Cómo explicaríais estos cambios en la población de mariposas geómetras?

Comentario: Aqui, la mayoria de las hipótesis se refieren simplemente a que las mariposas negras aumentan porque pasan desapercibidas y las claras disminuyen al ser comidas por los pájaros.

Algunos alumnos se refieren también a que algunas mariposas se vuelven negras al adaptarse al nuevo medio, pero abandonan sus concepciones al realizar la actividad 2, no solamente por el peso de la propia cuestión, sino también por el peso de los argumentos en contra defendidos por sus compañeros $y$ compañeras de clase.

\section{Actividad 2}

En 1956, el gobierno inglés promulga una ley para reducir la contaminación atmosférica. Desde entonces, la proporción de mariposas geómetras de color claro recogidas en áreas industriates ha vuelto a crecer.

¿Como se ha producido este aumento de las mariposas claras?

\section{Actividad 3}

Contrastad vuestras hipótesis con el siguiente documento:

Las investigactones realizadas han permitido confirmar que los pájaros encuentran y comen más mariposas oscuras que claras sobre las cortezas de los árboles cubiertas de líquenes, y más mariposas claras sobre cortezas oscuras.

Así, en una zona industrial, Ias mariposas oscuras tienen mayores probabilidades de sobrevivir y dejar descendencia. Al cabo de cierto tiempo aumentará, por lo tanto, la proporción de mariposas oscuras de una población.

Esto ayuda a explicar el cambio rápido sufrido por la poblactón de las mariposas geómetras en las ciudades industriales en los primeros años de la revolución industrial.

Pero, ¿qué está pasando ahora?
En el año 1956, después de la ley para reducir la contaminación atmosférica, la cantidad de mariposas claras de las zonas industriales ha vuelto a crecer. Las mariposas oscuras que ahora descansan en las cortezas claras de los arboles cubiertas de líquenes son mejor vistas por los pájaros. Así, se ha ido reduciendo su número y aumentando el de mariposas claras que pasan más desapercibidas.

Adaptación de un texto de British Museum (1981)/ Diputació de Barcelona (1982).- L'origen de les espécies.

Comentario: Este bloque de actividades resulta generalmente util e interesante, ya que la teoría propuesta por Darwin permite explicar muy bien los problemas planteados.

Es sabido que para "derrumbar" una teoría científica -lamarckista en el caso de los alumnos- no es sufiente demostrar que ésta no sirve para dar respuesta a determinados problemas, es preciso ver que existen otras teorías que si explican estos problemas.

\section{0. ¿PUEDE EXPLICARSE LA EVOLUCIÓN HUMANA POR LA TEORÍA DE LA EVOLUCIÓN POR SELEC. CIÓN NATURAL? ¿CÓMO SE ORIGINARON LAS MU- JERES Y LOS HOMBRES?}

Comentario: No se puede terminar el tema sin abordar la problemática de la evolución humana. Por este motivo proponemos unas breves actividades que tienen como objetivo hacer ver a los alumnos que es posible aplicar la teoria de la evolución por selección natural a la evolución humana.

Para contrastar las hipótesis de los alumnos, proponemos la leclura de unas páginas del libro de J. Monod, El Azar y la Necesidad. Editorial Tusquets, pp. 163-167.

\section{Actividad 1}

En la figura 1 se muestra una imagen imaginaria y el cráneo de un animal llamado procónsul que vivió sobre la Tierra hace unos 25.000 .000 de años. Su cráneo tenía unas características parecidas a las del hombre y la mujer actuales y también al de los monos.

En la figura 2 se puede ver la imagen de un Australopitecus que vivió hace 5.000 .000 de años y que se considera como el miembro más antiguo descubierto de la familia humana.

¿Cómo se podría explicar de acuerdo con la teoría de la selección natural de Darwin el origen de los seres humanos? Emitir hipótesis de trabajo.

\section{Actividad 2}

Contrastad vuestras hipótesis con la lectura del texto de J. Monod, El Azar y la Necesidad. 